\title{
Hospital-acquired acute kidney injury prevalence in in adults at a South African tertiary hospital
}

\author{
Kate Fenna ${ }^{1,2}$, Rajiv T Erasmus ${ }^{1}$, Annalise E Zemlin ${ }^{1}$
}

1. Division of Chemical Pathology, National Health Laboratory Service (NHLS) and Stellenbosch University, Tygerberg Hospital, Cape Town, South Africa.

2. Royal Surrey County Hospital, NHS Foundation Trust, District General Hospital, Surrey, United Kingdom.

\section{Emails:}

kate.fenna@nhs.net,rte@sun.ac.za, azemlin@sun.ac.za

\begin{abstract}
:
Background: Hospital Acquired Acute Kidney Injury (HA-AKI) prevalence has not been analysed in a South African setting. We investigated HA-AKI prevalence, using the KDIGO definition, with clinical characteristics and outcomes. The aim was to provide evidence for earlier treatment interventions to improve outcomes, such as recent UK NHS initiatives of automated electronic alerts in the laboratory information system.

Methods: Retrospective laboratory and clinical data was analysed for a 6-month period at Tygerberg Hospital, Cape Town. Serum creatinine results and clinical records were analysed and collated into gender and age group specific results.

Results: HA-AKI occurred in $6.2 \%$ of hospitalised patients for the period of analysis. The highest incident occurred in females aged 18-39 and males aged 40-59. The most common AKI stage reached was stage 1. HA-AKI increased length of stay by an average of 4.6 days and $20 \%$ of patients were readmitted at a later date with renal dysfunction.

Conclusion: AKI prevalence is significant and associated with adverse patient outcomes. Initiatives that allow front-line healthcare professionals to treat and manage AKI, such as introduction of automated electronic alerts, should be considered. Similar initiatives have been implemented in UK NHS hospitals with positive impacts.
\end{abstract}

Keywords: Acute kidney, injury prevalence, South African.

DOI: https://dx.doi.org/10.4314/ahs.v19i2.44

Cite as: Fenna K, Erasmus RT, Zemlin AE. Hospital-acquired acute kidney injury prevalence in in adults at a South African tertiary hospital. Afri Health Sci.2019;19(2): 2189-2197. https:/ / dx.doi.org/10.4314/ahs.v19i2.44

\section{Introduction}

A common complication affecting hospital in-patients worldwide is acute kidney injury (AKI) ${ }^{1-3}$. Characterised by rapid decline in kidney function and oliguria, it is associated with adverse long-term outcomes and high mortality. The Kidney Disease Improving Global Outcomes (KDIGO) clinical guideline states that $\mathrm{AKI}$ is defined and

\section{Corresponding author: \\ Annalise E Zemlin, \\ P.O. Box 19113, \\ Tygerberg, 7505, \\ South Africa, \\ Fax: (+27) 219384640 \\ Tel: (+27) 219384107 \\ Email: azemlin@sun.ac.za}

staged based on an increase in Serum Creatinine (SCr) concentration and reduction in urine output. The effects are both short and long-term and even a minor acute reduction in kidney function has an adverse prognosis. Several studies have confirmed that severity and duration of AKI determines outcome ${ }^{2,4,5}$. Although common and harmful, AKI is potentially preventable and treatable. There has been a global drive for earlier recognition and prevention strategies as this improves patient outcomes ${ }^{3}$. One strategy implemented by National Health Service (NHS) England was the introduction of a national algorithm to standardise the definition of $\mathrm{AKI}^{4,5}$. This algorithm was agreed in the United Kingdom (UK) in June 2014 and was integrated into the Laboratory Information Management Systems (LIMS) used by UK NHS hospitals. It identifies and stages real-time potential cases of

(C) 2019 Fenna et al. Licensee African Health Sciences. This is an Open Access article distributed under the terms of the Creative commons Attribution License (https://creativecommons.org/licenses/BY/4.0), which permits unrestricted use, distribution, and reproduction in any medium, provided the original work is properly cited. 
Hospital Acquired Acute Kidney Injury (HA-AKI) using the current laboratory results and previous laboratory results linked to the patient's records, sending results to patient management systems for urgent intervention, to ensure timely and consistent approaches. Data from earlier UK based studies on AKI prevalence rates, patient outcomes and predicted cost implications were a major driving force for such initiatives ${ }^{1,2}$. Therefore the data from this study may provide evidence for the introduction of similar strategies.

Overall global AKI incident rates are not well known and recent studies conducted in multiple countries have shown wide disparity in reported incident rates ${ }^{6}$. Previous 2014 UK based studies estimated that $14-20 \%$ of hospitalised patients developed AKI, 2.1\% of which is hospital-acquired, with a suggested $20-30 \%$ preventable ${ }^{1,6,7}$. It was also suggested that patients most at risk of developing AKI whilst in a UK hospital were aged $>70$, with mean age of $74.4^{1,6}$. The overall conclusions were that AKI is associated with a high number of in-hospital deaths with high healthcare $\operatorname{costs}^{1,8}$. The purpose of this study was to determine the prevalence of HA-AKI at Tygerberg Hospital, Cape Town, a less economically developed healthcare setting, to compare the prevalence with the UK study, and estimate the impact on both the patient and healthcare system.

\section{Methods and materials Study site}

A retrospective audit of laboratory and clinical records of finished hospital admissions at Tygerberg Hospital for a given 6 month period (01/12/2015-01/06/2016) was performed. Tygerberg Hospital is a tertiary hospital in Cape Town, South Africa and provides inpatient and outpatient care to public health-sector users ${ }^{9}$. It is a 1,400 bed multidisciplinary teaching hospital affiliated with Stellenbosch University. The National Health Laboratory Service (NHLS) Chemical Pathology laboratory at Tygerberg Hospital provides 24 hour diagnostic service. The NHLS is the preferred provider of pathology services to the public health-sector.

\section{Definitions}

HA-AKI incidence was estimated using the number of patients that developed biochemical markers indicative of AKI in line with the KDIGO definition whilst in hospital, out of the total number of inpatient admissions for the stated six month period. Patients admitted through
Accident and Emergency (A\&E) and hospital outpatient clinics were included. The analysis was restricted to patients aged $>18$ and excluded day case and maternity admissions and any patients with AKI on initial hospital presentation.

\section{Laboratory data collection}

Laboratory data for the specified dates and specific requesting locations (Tygerberg Hospital wards and outpatients only) was extracted from the LIMS used at Tygerberg Hospital (Disa*Lab), and collated anonymously into a Microsoft Excel spread sheet before subsequent analysis. This data included; patient age at referral, gender, unique medical record number, unique hospital folder number, serial serum creatinine $(\mathrm{SCr})$ results $(\mu \mathrm{mol} / \mathrm{L})$, serial serum urea results $(\mathrm{mmol} / \mathrm{L})$, serial electrolyte results $(\mathrm{Na}, \mathrm{K}, \mathrm{Cl})$.

The cohort was divided by gender to create two separate spread sheets; male and female. The results were then grouped according to the patient unique identifier in date order to allow serial results for each patient to be analysed. In order to include only patients that developed biomarkers indicative of AKI during their stay as an inpatient at Tygerberg Hospital, only patients that had a normal baseline SCr on admission, that increased in line with the KDIGO definition $>48$ hours post admission whilst under hospital care were included ${ }^{8}$. All patients with normal serial SCr results during their hospital stay or raised SCr on initial presentation were therefore excluded and the results removed. Patients with $>3$ serial SCr results that increased in line with the KDIGO definition of AKI during their hospital stay were included ${ }^{6,8}$. The two data sets (males and females) were then further sub-grouped into four age groups; 18-39, 40-59, 60-79, 80+. This created a total data set of 8 sub-groups on separate spread sheets for analysis.

\section{Clinical data collection}

Hospital admission data, cause of the AKI, length of hospital stay and readmission data was analysed using the electronic medical record system available at Tygerberg Hospital. Due to time restraints, a sample population cohort was used. Medical records for a cohort of 10 males and 10 females from each of the 4 age groups, creating a total of 80 patients, was analysed in detail. All data was extracted anonymously into an Excel spread sheet for subsequent analysis. The total number of hospital in-patient admissions for the specified dates was accessed from the hospital admissions record office.

African Health Sciences Vol 19 Issue 2, June, 2019 


\section{Laboratory analysis}

Laboratory analysis of SCr was performed on a Siemens Advia ${ }^{\circledR} 1800$ automated analyser at Tygerberg Hospital Chemical Pathology laboratory using the Jaffe method. The reference ranges used for SCr analysis were taken from the standard operating procedure (SOP) at Tygerberg Hospital NHLS Chemical Pathology Laboratory CHE0410. This SOP states gender specific reference ranges for SCr measurement in patients aged $>18$ are as follows; Males 64-104 $\mu \mathrm{mol} / \mathrm{L}$, Females 49-90 $\mu \mathrm{mol} / \mathrm{L}$. Serial SCr results for each patient were analysed to determine AKI staging and where possible clinical records were analysed for recorded urine output. These results were also analysed to determine renal recovery post-AKI before discharge. Serum U\&E were analysed for further evidence of decline in renal function. Clinical records for a sample population of each sub-group were analysed to determine initial diagnosis, hospital stay duration and where possible patient outcome in terms of increased length of stay and return hospital visit with abnormal renal function.

\section{Numerical analysis}

To analyse AKI prevalence for each sub-group, in relation to total in-patients, data from Tygerberg Hospital admission report was accessed. As an estimate of associated HA-AKI risk factors clinical records were analysed to determine initial presentation and diagnosis for a sub-set of 80 patients; 10 males and 10 females in each of the 4 age groups. Patients were selected based on age (to ensure the whole age range was represented) and completeness of biochemistry results available. To provide an insight into the impact on patient outcomes and hospital resources, clinical records for the same 80 patients were analysed to determine total length of hospital stay, length of stay after AKI diagnosis and recurrent admission since the initial HA-AKI with abnormal renal function.

\section{Results}

The total number of inpatient hospital admissions for the given 6 month time period was 18,781. Using retrospective analysis of laboratory and clinical records, total number of patients aged $>18$ with a diagnosis of AKI was 2,760 and therefore total prevalence of AKI at Tygerberg Hospital (community and hospital acquired) was estimated to be $14.7 \%$. The total number of patients aged $>18$ that developed HA-AKI was 1,165. It was therefore concluded that total HA-AKI prevalence for Tygerberg Hospital for the given 6 month period was $6.2 \%$. The characteristics of the 1,165 patients used in this study are shown in table 1 .

Patients were sub-grouped according to gender and age group. Of the 1,165 cases of HA-AKI, 689 were female $(59.1 \%)$ and $476(40.9 \%)$ were males. Analysis of age group incidence showed highest prevalence occurred in females aged 18-39 (294 cases) and males aged 40-59 (195 cases). Results for the total number of patients that developed HA-AKI for each of the 8 sub-groups are shown in figure 1. 
Table 1. Characteristics of patients with hospital acquired acute kidney injury $(\mathbf{N}=1165)$

\begin{tabular}{ll}
\hline Variable & Study Cohort \\
\hline Age(years)(mean $\pm \mathrm{SE})$ & $46 \pm 0.4$ \\
Gender (male:female) & $1: 1.45$ \\
Total hospital stay (days) (mean $\pm \mathrm{SE})$ & $10.8 \pm 2.1$ \\
Study entry serum creatinine $(\mu \mathrm{mol} / \mathrm{L})($ mean $\pm \mathrm{SE})$ & $55.9 \pm 0.15$ \\
Study entry serum urea (mmol/L) (mean $\pm \mathrm{SE})$ & $4.4 \pm 0.01$ \\
Peak serum creatinine $(\mu \mathrm{mol} / \mathrm{L})($ mean $\pm \mathrm{SE})$ & $380.1 \pm 4.48$ \\
Peak serum urea (mmol/L) (mean $\pm \mathrm{SE})$ & $20.43 \pm 0.17$ \\
Reason forhospital admission in select sub-group: & \\
(total of 80 patients) & \\
$\quad$ Infection & 37 \\
$\quad$ Hypertension & 14 \\
$\quad$ Planned surgery & 11 \\
Immunocompromised (SLE, AIDS, & 8 \\
Immunotherapy, Malignancy) & 6 \\
Nausea and vomiting & 4 \\
Fall & \\
\hline
\end{tabular}

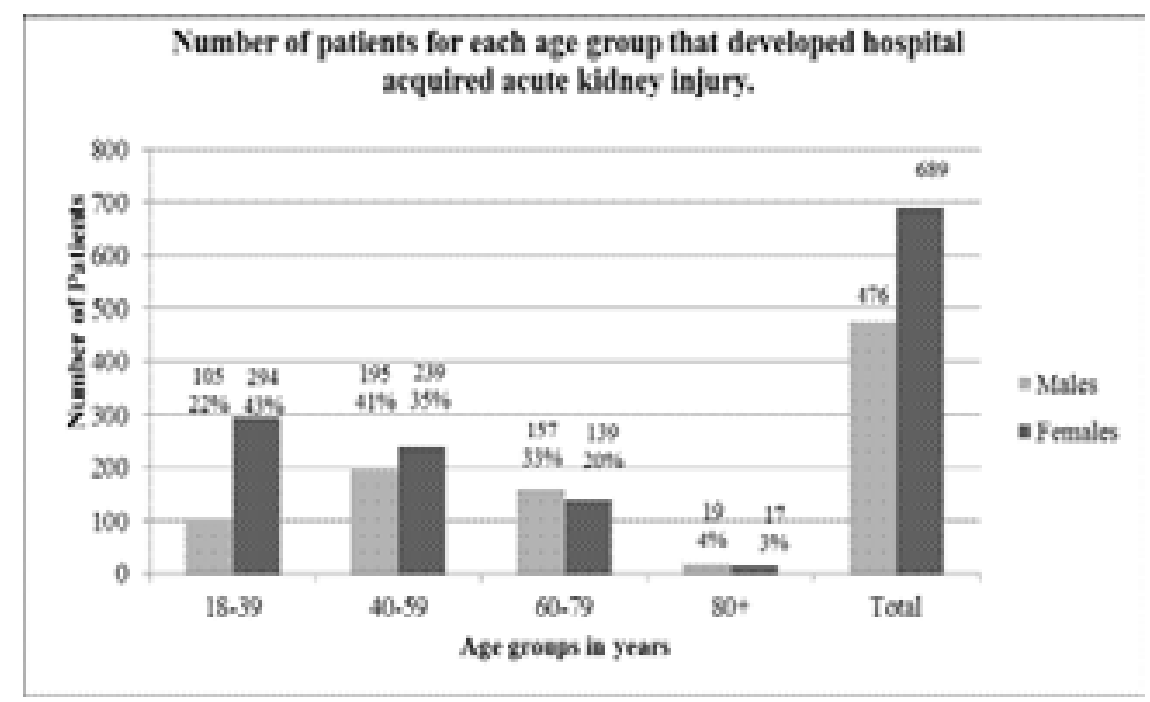

Figure 1 - the total number of patients for each age group of males and females that developed biochemical markers indicative of acute kidney injury, in line with the KDIGO definition of AKI, during their stay as an in-patient at tygerberg tertiary hospital. 
AKI staging was determined using the KDIGO AKI definition of changes in serum creatinine over time, using the lowest serum creatinine measurement from the same admission as baseline, and highest SCr reached during the same admission for severity staging. The results of which, as expected, showed the most common AKI stage reached was an AKI stage 1, with some patients progressing to a stage 2 or 3 . The results for AKI staging in females are shown in figure 2 , and AKI staging in males in figure 3.

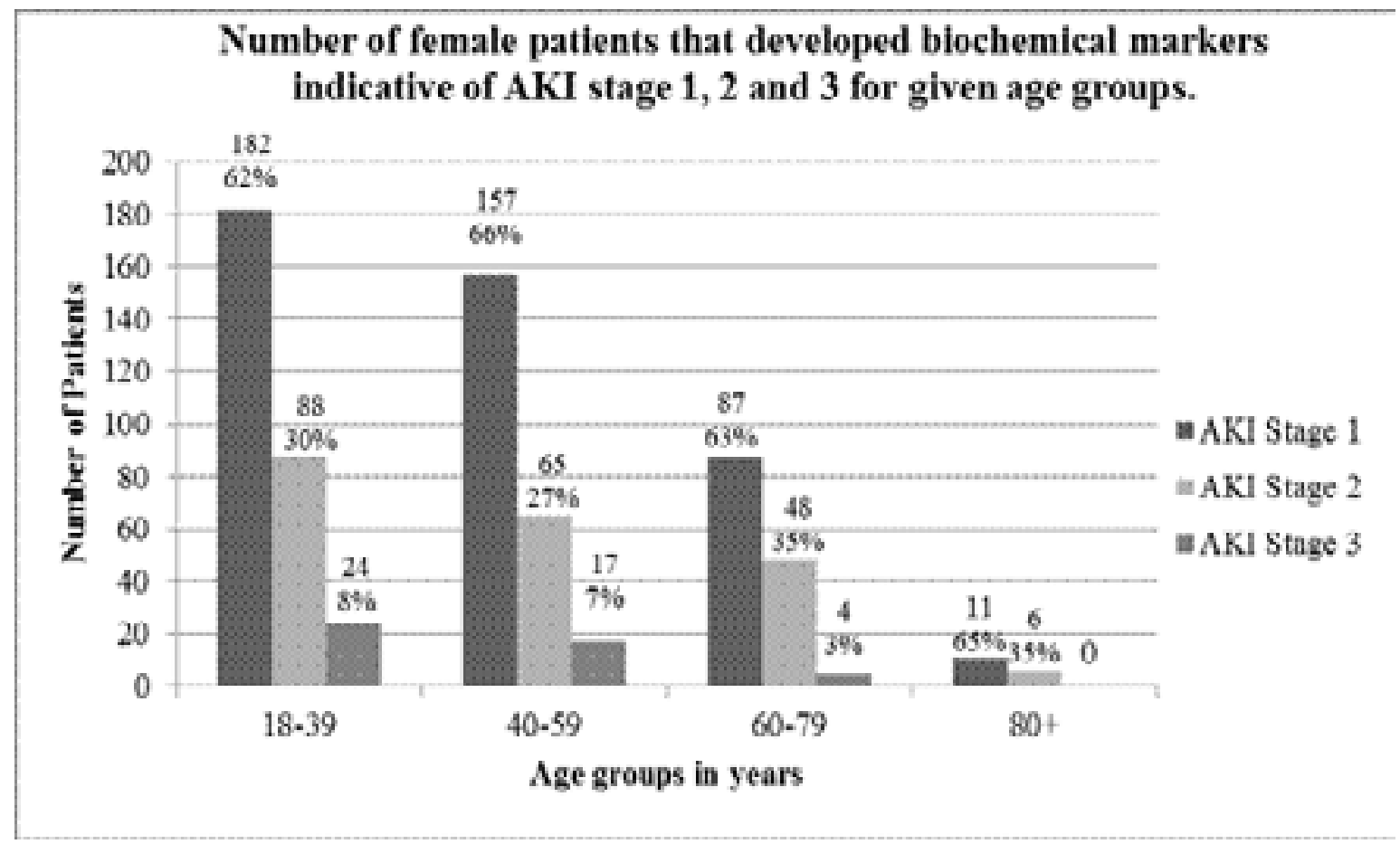

Figure 2 - The number of female patients for each age group that developed biochemical markers indicative of acute kidney injury stage 1, 2 and 3, in line with the KDIGO definition of AKI, during their stay as an in-patient at Tygerberg Tertiary Hospital. The numbers above each bar are the exact number of patients; the percentage value is the percentage of patients that developed stage 1, 2 and 3 for the given age group to the nearest whole number. 


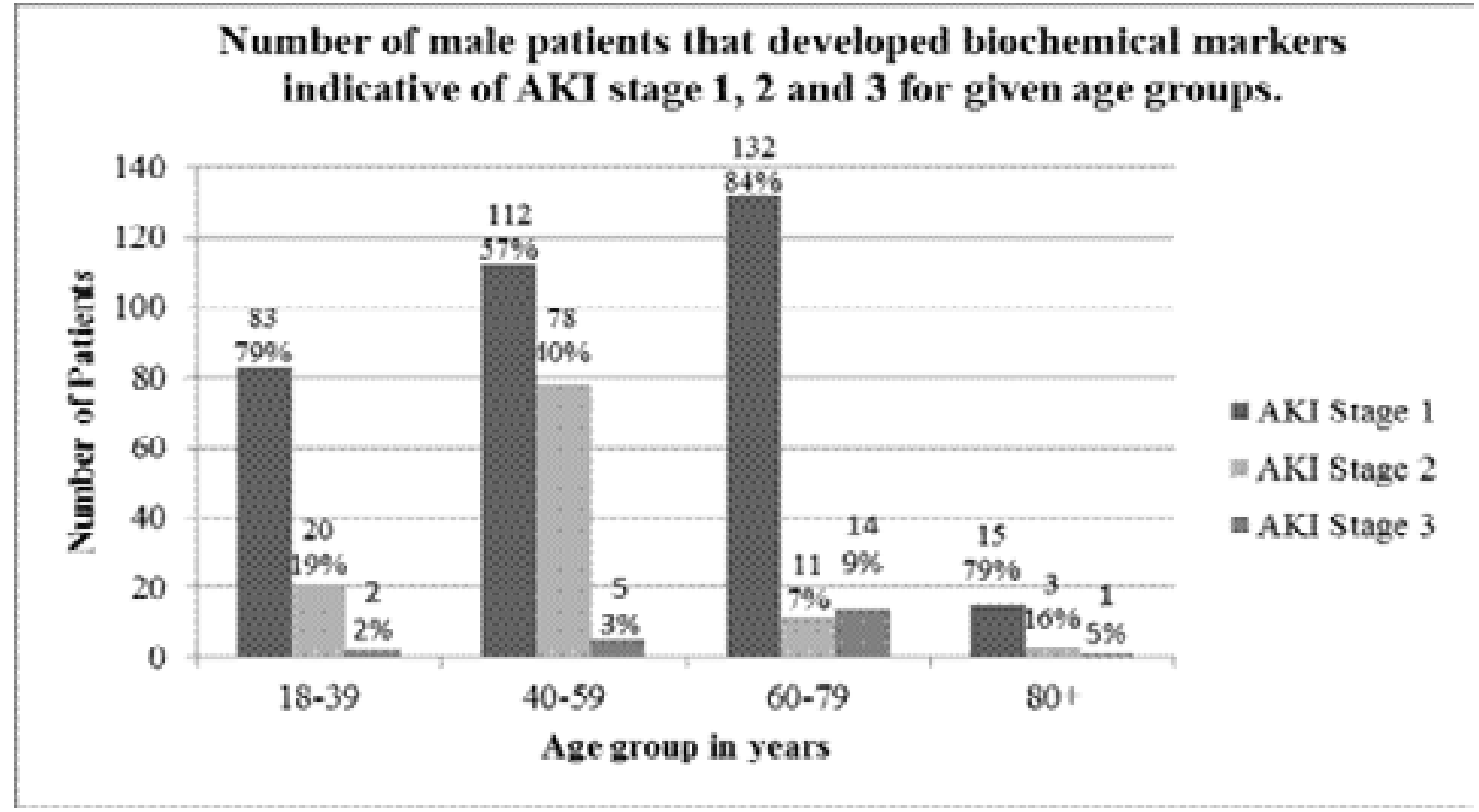

Figure 3 - The number of male patients for each age group that developed biochemical markers indicative of acute kidney injury stage 1, 2 and 3, in line with the KDIGO definition of AKI, during their stay as an in-patient at Tygerberg Tertiary Hospital. The numbers above each bar are the exact number of patients; the percentage value is the percentage of patients that developed stage 1,2 and 3 for the given age group to the nearest whole number.

Initial diagnosis at hospital admission was analysed for a sub-set of 10 patients ( 5 females and 5 males) that developed HA-AKI from each of the 4 age groups, creating a subset of 80 patients. This showed the most common initial presentation and therefore risk factor for HA-AKI was infection, the second hypertension and third a surgical procedure. The initial diagnosis at hospital admission for the patient cohort used is summarised in table 1. Total length of hospital stay and length of stay post HA-AKI diagnosis was also analysed. This showed the mean increased length of stay after HA-AKI onset for the patient subset analysed was 4.6 days (mean $=4.6, \mathrm{SE}=0.79$ ). The impact of HA-AKI on increased length of stay has both negative social and economic impacts.

Clinical records for separate patient admissions following the initial admission were also analysed, as well as laboratory results and clinical records for evidence of abnormal renal function following the HA-AKI incident. This analysis provided an insight into long-term patient outcomes. The results showed that of the subset of 80 patients an- alysed, 25\% were readmitted to hospital at a later date (20 out of 80 patients), with impaired renal function. The highest rate of readmission was observed in females aged 40-59 and males 60-79.

\section{Discussion}

The aim of this study was to determine the prevalence of HA-AKI at Tygerberg Hospital, compare this to the previously published prevalencein the $\mathrm{UK}$ and estimate the impact of HA-AKI on the patient and healthcare system.

AKI is a common and serious health complication affecting a significant number of hospital in-patients ${ }^{1,6,7}$. However, it is easily preventable and potentially treatable if at risk patients are diagnosed early and monitored ${ }^{1}$. Recently there has been a global drive in earlier recognition and prevention strategies to improve patient outcomes ${ }^{6}$. A key initiative implemented in UK NHS hospital laboratories is the introduction of automated electronic alerts in the LIMS ${ }^{5,8,10}$. Our study has provided an insight into rates of $\mathrm{HA}-\mathrm{AKI}$ in a SA hospital, and has contributed 
to the global need for epidemiology studies. The results found that $6.2 \%$ of patients admitted to Tygerberg Hospital for the given 6 month period developed HA-AKI. Similar studies have been completed in other global locations, with rates of HA-AKI ranging from 1-7\% $0^{1,3,11-15}$. A 2014 UK based study with comparable methodology found HA-AKI prevalence to be 2.1\% ${ }^{1}$. An earlier 1996 study also using comparable methods based in Chicago, USA, found that AKI developed in $7.2 \%$ of hospitalised patients, with medications, surgery and radiographic contrast media use being the most common causes ${ }^{11}$.The significant increase in HA-AKI prevalence found in the 1996 USA based study compared to the 2014 UK study may be due to medical advances and increased awareness. The results of our study, which showed HA-AKI prevalence to be similar to the 1996 USA based study $(6.2 \%$ and $7.2 \%$ respectively), may reflect the current comparatively naïve status of the SA healthcare system.

The data from this study identified the most at risk patient cohort for the development of HA-AKI were females aged 18-39 and males aged 40-59, with average age of onset of 44 in females and 51 in males. A similarly designed 2014 study conducted in South Western Ghana looking at AKI incidence from retrospective laboratory data found highest prevalence in the age group 20-39, with females at increased risk at a younger age ${ }^{12}$. A 2016 multicentre study of AKI prevalence in ICU patients in Kinshasa, the Democratic Republic of Congo found the average AKI patient age of $51.9^{13}$. Both of these independent studies therefore support our results. However, when compared to UK based studies, where average age of AKI onset is $\sim 74.4$, this is a significantly younger age. The World Health Organization (WHO) 2015 statistics state life expectancy at birth for SA is 66 for females and 59 for males ${ }^{14}$. Comparatively, the UK life expectancy at birth is 83 for females and 79 for males ${ }^{15}$. Therefore the relationship of HA-AKI onset later in life, closer to respective life expectancy is reflected in both the UK study and the African based studies, and is well supported by literature that shows a risk factor for $\mathrm{AKI}$ is increased age $\mathrm{e}^{1,12,13}$. The highest prevalence of HA-AKI in females aged 1839 found in this study reflects child bearing age and the well documented association of pregnancy and $\mathrm{AKI}^{6}$. Although patient samples from maternity were not included, previous history of pregnancy and child birth was not included in the clinical records analysed and therefore the high prevalence of HA-AKI in females aged 18-39 may reflect the increased risk of prior child bearing. It is widely recognised that AKI epidemiology differs in developing vs. developed countries in many important ways ${ }^{3,4}$. A previous 2008 study found that infections, nephrotoxins, and obstetric and surgical complications are amongst the major causes of AKI in Africa, with the most common risk factors being severe immune suppression and opportunistic infection ${ }^{4,14,15}$. It was also found that HIV-1-infected patients with AKI have an increased risk of in-hospital mortality 4. Due to time limitations of our study, clinical details on infections and HIV status were not analysed, however it is recognised that these are important risk factors. Another key documented difference is the age of onset. In developed countries AKI predominantly occurs in the elderly, and thus increasing age is a significant risk factor $^{3,4}$. On the other hand, in developing countries AKI is considered a disease of the young ${ }^{4}$. The results of this study therefore further support the increased prevalence of AKI in younger patients in terms of actual years, but not younger in terms of stage of life expectancy.

The analysis of subsequent hospital admissions in a subset of patients identified that $20 \%$ of the patients had been readmitted with laboratory results suggestive of abnormal renal function; with the highest re-admission observed in females aged 40-59 and males aged 60-79. In the $80+$ cohort for both males and females, only $1 \%$ of patients was readmitted; perhaps due to mortality as this is well above the current SA life expectancy of 66 and 59 for females and males respectively ${ }^{14}$. A recent 2016 publication of a systematic review and meta-analysis of hospital readmissions following an AKI event found that hospital readmission rates were $21 \%{ }^{17}$. The results of this study closely reflect that, with a readmission rate of $20 \%$. Ideally, in order to directly compare these statistics this study should be extended to a 12 month period to account for seasonal changes. However, the readmission rate of $20 \%$ is significant, which emphasises the importance and burden of AKI on medical resources; which may have been preventable, further highlighting the importance of rapid AKI diagnostics and early kidney-sparing initiatives.

The development of AKI on average increased hospital stay in our cohort by 4.6 days, which has significant economic impacts on the total cost of patient care. Previous studies have reported similar supportive findings. 
A 2011 American study looking at HA-AKI prevalence and outcomes concluded that HA-AKI increases hospital stay by on average 4 days ${ }^{17}$. This is a similar and supportive finding. This data should therefore provide evidence and encouragement for early diagnostic and prevention initiations. Patients that developed HA-AKI from all in-patient locations at Tygerberg Hospital were included in this study. It is however well recognised that Intensive Care Unit (ICU) patients are most at risk of AKI development, particularly within a low resource setting ${ }^{6,13,17,18}$. A recent 2016 multicentre study conducted in 7 ICU settings from Kinshasa, the Democratic Republic of Congo found that the cumulative incidence of AKI in ICU patients was $52.7 \%{ }^{18}$. This therefore identifies a particularly vulnerable patient cohort that could benefit from earlier diagnostic and intervention initiatives.

AKI is a challenging global burden, with significant health and cost implications. This therefore emphasises the importance of finding and introducing improved diagnostic and treatment interventions. A strategy recently implemented in all UK NHS hospitals by NHS England, is the introduction of a national algorithm to standardise AKI definition and allow real time identification of potential cases of AKI from laboratory data, sending results to patient management systems for urgent intervention ${ }^{4,5}$. This allows AKI to be detected and staged based on changes in biochemical markers in line with national guidelines and alerts the healthcare professionals to act accordingly in a timely and consistent approach. It should be noted that this automated electronic alert is only an aid to prompt early recognition of AKI and should not be relied upon to detect AKI without close inspection of serial blood test results and clinical evaluation ${ }^{1,4,5}$. AKI is a clinical diagnosis based on clinician review of serial blood test results in the clinical context of the individual patient and therefore this standard should be maintained. However, in a healthcare system such as that in SA where there is a significantly low ratio of doctors to patients in the public setting, but an acceptable ratio of healthcare professionals to patients, an initiative such as this could allow early awareness of a patient at risk of or that has developed an $\mathrm{AKI}^{19,20}$.

Our study had several limitations. Due to the medical record system at Tygerberg Hospital, identifying the initial diagnosis and cause of AKI was extremely difficult. Handwritten notes are scanned into an electronic system, and are often incomplete and difficult to read. Due to the absence of a national identity system in SA it is often difficult to see a patients full clinical history or follow a patient for long term outcomes once discharged from a hospital. We therefore were unable to access treatment, outcome or mortality data.

Unfortunately this study was performed over a short time term (KF's Elective) and therefore a repeat study over a 12 month period to include potential seasonal trends would be ideal. Our study was conducted in one Tertiary Hospital; ideally repeat at multiple hospitals for a true representation of the SA healthcare system. Infection and HIV status were not included in this study, but have been previously identified as a major cause of $\mathrm{AKI}^{17,13,16}$.

\section{Conclusion}

HA-AKI prevalence at Tygerberg Tertiary Hospital for the given 6 month period was $6.2 \%$. The most at risk patients were found to be females aged 18-39 and males aged 40-59; with mean age of onset of 46 years. The most common HA-AKI staging reached was AKI stage 1. The onset of HA-AKI increased hospital length of stay by 4.6 days on average, and had a $20 \%$ readmission rate with abnormal renal function. The most common initial presentation was an infection, followed by hypertension and then surgery. Public SA hospitals have a significantly low doctor to patient ratio $(0.8: 1000)$ but an acceptable healthcare professional to patient ratio (2.9:1000) and therefore interventions that will bring AKI to the attention of healthcare professionals sooner should be investigated $^{19,20}$. One such intervention recently implemented by all UK NHS hospitals is the incorporation of a national algorithm into the LIMS generating automated electronic alerts for urgent response. Such initiatives may be considered for implementation into the SA LIMS. The LIMS used at Tygerberg Hospital is TrakCare. The Association of Clinical Biochemistry (ACB) national meeting statement to discuss E-Alerts for AKI held in July 2013 show a TrakCare representative was absent, but incorporation of this algorithm is technically possible ${ }^{10}$. AKI is a significant social and economic health burden and therefore 
any interventions to improve early diagnosis and treatment should be considered.

\section{Acknowledgements}

This study was conducted at Tygerberg Hospital by KF on a self-funded Elective visit.

We would like to thank Mr. W Kleinhans, for this help with data acquisition and Dr. N Naidoo for this help with the access to electronic medical records.

\section{Ethical consideration}

The study was approved by the Stellenbosch University Ethics Committee (HREC \#N16/09/106). A waiver of consent was approved due to the nature of the study and the study was performed according to the Declaration of Helsinki. Medical records were accessed for a subset of patients who had specifically had blood samples sent to the laboratory for clinical interpretation of renal function. Only the authors accessed these records and anonymity was maintained at all times.

\section{References}

1. Kerr M, Bedford M, Matthews B, O'Donoghue D. The economic impact of acute kidney injury in England. Nephrol Dial Transplant. 2014;29(7):1362-1368.

2. Coca SG, King JT, Rosenthal R, Perkal MF, Parikh CR. The duration of postoperative acute kidney injury is an additional parameter predicting long-term survival in diabetic veterans. Kidney Int. 2010;78(9):926-933.

3. Naicker S, Aboud O and Gharbi MB. Epidemiology of acute kidney injury in Africa. Semin Nephrol. 2008;28(4):348-353.

4. Hill R, Selby NM. Acute kidney injury warning algorithm best practice guidance. Think Kidneys National Programme 2014; 2-10.

5. National Health Service England. Acute kidney injury (AKI) algorithm, https://www.england.nhs.uk/patientsafety/akiprogramme/aki-algorithm (2014, accessed 12 September 2016).

6. Wonnacott A, Meran S, Amphlett B, Talabani B, Phillips A. Epidemiology and outcomes in community-acquired versus hospital-acquired AKI. Clin J Am Soc Nephrol 2014;9(6): 1007-1014.

7. Lewington AJ, Cerdá J, Mehta RL. Raising awareness of acute kidney injury: a global perspective of a silent killer. Kidney Int 2013;84(3):457-467.

8. Kidney Internal Supplements, www.kdigo.org/clinical_
practise_guidelines/pdf/CKD/KDIGO_2012_CKD_ GL, (2012, accessed 12 September 2016).

9. Western Cape Government. Tygerberg Hospital, www. westerncape.gov.za/your_gov/153 (2015, accessed 12 September 2016).

10. The Association for Clinical Biochemistry and Laboratory Medicine. E-Alerts for GFR Meeting Derby, www. acb.org.uk/docs/E-Alerts_for_AKI_meeting_statement (2013, accessed 12 September 2016).

11. Nash K,Hafeez A, Hou S. Hospital-acquired renal insufficiency. Am J Kidney Dis 1996; 39(5): 930-936.

12. Ephraim E,Darkwah KO, Sakyi SA, Ephraim M, Antoh EO, Adoba P. Assessment of the RIFLE criteria for the diagnosis of acute kidney injury; a retrospective study in South-Western Ghana. BMC Nephrol 2016; 17: 87-99. 13. Broce JC, Price LL, Liangos O, Uhlig K, Jaber BL. Hospital-acquired acute kidney injury: an analysis of nadir-to-peak serum creatinine increments stratified by baseline estimated GFR. Clin J Am Soc Nephrol 2011;6(7):15561565.

14. World Health Organization. WHO South Africa, http://www.who.int/countries/zaf/en/, (2015 accessed 12 September 2016).

15. World Health Organization. WHO United Kingdom, http://www.who.int/countries/zaf/en/, (2015 accessed 12 September 2016).

16. Siribamrungwong M, Chinudomwong P. Relation between acute kidney injury and pregnancy-related factors. Journal of Acute Disease 2016;5(1):22-28.

17. Zhang T, Hu J, Song N, Yu X, Xu X, Jia P, et al. Hospital readmissions after acute kidney injury: a systematic review and meta-analysis. Int J Clin Exp Med 2016;9(7):13456-13465.

18. Masewu S,Makulo JR, Lepira F, Amisi EB, Sumaili $\mathrm{EK}$, Bukabau J, et al. Acute kidney injury is a powerful independent predictor of mortality in critically ill patients: a multicenter prospective cohort study from Kinshasa, the Democratic Republic of Congo. BMC Nephrol 2016;17: 110-118.

19. World Health Organization: Spotlight on health workforce statistics is a series of fact files produced by the Department of Human Resources for Health, World Health Organization, www.who.int/hrh/statistics/Spotlight_6_Nov2008_benchmarking.pdf (2008, accessed 12 September 2016).

20. UK Renal Registry: The Acute Kidney Injury Programme,www.renalreg.org/projects/the-acute-kidney-injury-aki-programme (2012, accessed 12 September 2016). 